\title{
A Note on Independent Sets in Graphs with Large Minimum Degree and Small Cliques
}

\author{
Jeremy Lyle \\ Department of Mathematics \\ The University of Southern Mississippi \\ Mississippi, U.S.A. \\ samuel.lyle@usm.edu
}

Submitted: Nov 13, 2013; Accepted: May 15, 2014; Published: May 22, 2014

Mathematics Subject Classifications: 05C

\begin{abstract}
Graphs with large minimum degree containing no copy of a clique on $r$ vertices $\left(K_{r}\right)$ must contain relatively large independent sets. A classical result of Andrásfai, Erdős, and Sós implies that $K_{r}$-free graphs $G$ with degree larger than $((3 r-7) /(3 r-$ 4) $)|V(G)|$ must be $(r-1)$-partite. An obvious consequence of this result is that the same degree threshold implies an independent set of order $(1 /(r-1))|V(G)|$. The following paper provides improved bounds on the minimum degree which would imply the same conclusion. This problem was first considered by Brandt, and we provide improvements over these initial results for $r>5$.
\end{abstract}

\section{Introduction}

In one of the founding results in extremal graph theory, Turán [Tur41] determined the value of ex $\left(n, K_{r}\right)$, the number of edges which imply that a graph on $n$ vertices contains a copy of $K_{r}$. The extremal graphs are the classical Turán graphs, which, for $n$ divisible by $r-1$, are composed of $r-1$ equally-sized independent sets such that two vertices are adjacent if and only if they are contained in different independent sets. Such graphs are regular with degree $\delta=((r-2) /(r-1)) n$.

In fact, any $K_{r}$-free graph with minimum degree "almost as large" as Turán graphs must share some of the same properties. In particular, Andrásfai, Erdős, and Sós [AES74] showed that for a $K_{r}$-free graph $G$ (on $n$ vertices), $\delta(G)>((3 r-7) /(3 r-4)) n$ implies that $\chi(G) \leqslant r-1$. An extension of this problem is the chromatic threshold problem for a general graph $H$, which is to determine the threshold on the minimum degree such that there is a bound (as a function of only $H$ ) on the chromatic number. Recently, good 
results have been obtained regarding triangle-free graphs (e.g., [Tho02, Łuc06, BTar]), and the general problem has been solved for all $H\left[\mathrm{ABG}^{+} 13\right]$.

One can interpret these results as follows: For a suitably large lower bound on the minimum degree, forbidding a copy of $K_{r}$ must result in a large independent set. This interplay between the density of $G$, the clique number of $G$, and the independence number of $G$ is well-studied; it is the classical Ramsey-Turán theory. Let $\mathbf{R T}(n, H, f(n))$ denote the maximum number of edges in an $H$-free graph on $n$ vertices containing no independent set of order $f(n)$. For instance, a construction of Bollobás and Erdös [BE76] and an upper bound of Szemerédi [Sze72], combined to give

$$
\mathbf{R T}\left(n, K_{4}, o(n)\right)=(1+o(1)) \frac{n^{2}}{8} .
$$

However, not much attention has been paid to the problem of determining exact bounds on the independence number of $K_{r}$-free graphs with large minimum degree. This is somewhat of a compromise between what is typically considered in Ramsey-Turán theory and the structural results extending the work of Andrásfai, Erdős, and Sós. In [Bra01], Brandt first considered this problem, and raised the following question, which is quite appealing due to its connection with both the results of [AES74] and the classical result of Turán. Let $\alpha(G)$ denote the independence number of a graph $G$.

Problem 1. Determine the value of $c$ such that for any $K_{r}$-free graph $G$ with $\delta>c n$,

$$
\alpha(G) \geqslant \frac{1}{r-1} n
$$

It was conjectured in [Bra01] that the correct value of $c$ is $c=(5 r-11) /(5 r-5)$, with the graphs described below to demonstrate the possible sharpness of this value.

The join of two graphs $G$ and $H$, denoted $G \vee H$, is defined by taking the disjoint union of the vertex sets from $G$ and $H$, i.e., $V(G \vee H)=V(G) \cup V(H)$ such that the subgraph induced by $V(G)$ is $G$, the subgraph induced by $V(H)$ is $H$, and $G \vee H$ also contains all edges of the form $(v, w)$, where $v \in V(G)$ and $w \in V(H)$. For $r$ odd, choose $\ell=(r-1) / 2$, and consider the graph

$$
H=\vee_{i=1}^{\ell} C_{5},
$$

where each vertex in a copy of $C_{5}$ can be replaced with an independent set of $b$ vertices.

For $r$ even, choose $\ell=(r-2) / 2$, and consider the graph

$$
H=\vee_{i=1}^{\ell} C_{5} \vee I,
$$

where each vertex in a copy of $C_{5}$ can be replaced with an independent set of $b$ vertices, and $I$ is an independent set of $a$ vertices, where $a=(5 / 2) b-k$, for some $k>0$ such that $l \ll k$.

Brandt showed that the conjecture holds for $r=4$ and $r=5$, and was able to prove a bound for general $r$. 
Theorem 2 (Brandt [Bra01]). Let $G$ be a $K_{r}$-free graph $(r \geqslant 4)$ on $n$ vertices.

1. If $r=4$, and $\delta(G) \geqslant(3 / 5) n$, then $\alpha(G) \geqslant(1 / 3) n$.

2. If $r=5$, and $\delta(G) \geqslant(7 / 10) n$, then $\alpha(G) \geqslant(1 / 4) n$.

3. If $r>5$, and

$$
\delta(G) \geqslant \frac{r^{2}-5}{r^{2}+r-2} n=\left(\frac{r-3}{r-2}+\frac{4}{(r-2)(r+2)(r-1)}\right) n,
$$

then $\alpha(G) \geqslant(1 /(r-1)) n$.

For a quick comparison, the conjectured bound can be written as

$$
\delta(G) \geqslant \frac{5 r-11}{5 r-5} n=\left(\frac{r-3}{r-2}-\frac{r-7}{5(r-1)(r-2)}\right) n .
$$

\section{Results}

Our contribution is to prove a "bootstrapping lemma", which will allow us to give an improvement on Theorem 2 for $K_{r}$-free graphs where $r \geqslant 6$, and additional structural information in the cases $r=4$ and $r=5$. In particular, we prove the following result (conjectured in [Bra01]) regarding $K_{4}$-free graphs which cannot be decomposed as the join of a triangle-free graph and an independent set.

Theorem 3. Let $G$ be a maximal $K_{4}$-free graph such that $\delta(G) \geqslant(4 / 7) n$. If $G$ is not the join of an independent set and a triangle-free graph, then $\alpha(G) \geqslant 4 \delta-2 n$.

As a result, we obtain an alternative proof of the bound in Theorem 2 in the case where $r=4$.

For the case $r=5$, we can prove a similar result regarding large independent sets in $K_{5}$-free graphs which cannot be decomposed as a join of either a $K_{4}$-free graph and an independent set, or as two triangle-free graphs.

Theorem 4. Let $G$ be a maximal $K_{5}$-free graph such that $\delta(G) \geqslant(11 / 16) n$. If $G$ is not the join of two triangle-free graphs, then

$$
\alpha(G) \geqslant \min \left\{3(3 \delta-2 n), \frac{1}{6}\left(20 \delta-\frac{25}{2} n\right), \frac{2}{3}(2 \delta-n)\right\} .
$$

As in the case of $K_{4}$-free graphs, we obtain an alternative proof on the bound of the minimum degree of a $K_{5}$-free graph on $n$ vertices which forces an independent set of order $(1 / 4) n$. In addition, bounds on the minimum degree which force an independent set of order $(1 / 5) n$ are also demonstrated.

Corollary 5. Let $G$ be a maximal $K_{5}$-free graph. 


\begin{tabular}{|l|l|l|l|l|l|}
\hline$r$ & 6 & 7 & 8 & 9 & 10 \\
\hline \hline Conjecture & $\frac{19}{25}=.76$ & $\frac{4}{5}=.8$ & $\frac{29}{35} \approx .829$ & $\frac{17}{20}=.85$ & $\frac{13}{15}=.8 \overline{6}$ \\
\hline Theorem 2 & $\frac{31}{40}=.775$ & $\frac{22}{27}=. \overline{814}$ & $\frac{59}{70} \approx .843$ & $\frac{19}{22}=.8 \overline{63}$ & $\frac{95}{108} \approx .880$ \\
\hline Theorem 6 & $\frac{61}{80}=.7625$ & $\frac{121}{150}=.80 \overline{6}$ & $\frac{211}{252} \approx .837$ & $\frac{337}{392} \approx .860$ & $\frac{505}{576} \approx .877$ \\
\hline
\end{tabular}

Table 1: Comparison of the conjectured thresholds with Theorems 2 and 6 .

1. If $\delta \geqslant(7 / 10) n$, then $\alpha(G) \geqslant(1 / 4) n$.

2. If $\delta \geqslant(31 / 45) n$, then $\alpha(G) \geqslant(1 / 5) n$.

Finally, we obtain a bound on the minimum degree of a $K_{r}$-free graph on $n$ vertices which forces an independent set of order $(1 /(r-1)) n$. This provides an improvement over Theorem 2 for all $r>5$.

Theorem 6. Let $G$ be a $K_{r}$-free graph $(r \geqslant 4)$. If

$$
\delta(G) \geqslant \delta_{r} n:=\left(\frac{r-3}{r-2}+\frac{1}{(r-2)^{2}(r-1)}\right) n,
$$

then $\alpha(G) \geqslant(1 /(r-1)) n$.

As a guide, Table 1 provides a comparison between the minimum degree bounds in Theorems 2 and 6 , as well as the conjectured bound.

\section{A General Lemma for $K_{r}$-free graphs}

To start with, the following lemma can be used to magnify weaker lower bounds on the independence number or obtain information regarding the structure of $K_{r}$-free graphs. The general idea is to examine a clique of order $K_{r-1}$ contained outside of an independent set $I$. Either vertices in $I$ are adjacent to the clique in a "nice", well-distributed manner, and a graph can be constructed which places an upper bound on $\alpha(G)$, or there is a large $K_{k}$-free subgraph which arises as the common neighborhood of a suitably chosen clique.

Given a $K_{r}$-free graph $G$ and an independent set $I$, we define a sunflower subgraph $S G_{r}$ as follows: First, take a clique of order $r-1$ (the "head" of the sunflower) which is disjoint from $I$. Then take $r-1$ vertices in $I$, each corresponding to (and adjacent to) one of the $r-1$ subsets of vertices of order $r-2$ in this clique. The presence of such a subgraph can be used to provide an upper bound on the order of an independent set.

Bootstrapping Lemma. Let $G$ be a maximal $K_{r}$-free graph with $n$ vertices.

One of the following statements regarding $G$ must be true.

1. $G$ is the join of an independent set and a $K_{r-1}$-free graph. 
2. For some $k$ such that $2 \leqslant k \leqslant r-2$, there is a set $U_{k} \subset V(G)$ such that the graph induced by $U_{k}$ is $K_{r-k}$-free, and

$$
\left|U_{k}\right| \geqslant k \delta-(k-1) n+\frac{k-1}{k} \alpha(G) .
$$

3. The value of $\alpha(G)$ is bounded above by

$$
\alpha(G) \leqslant 2(n-\delta)-\frac{2}{r-2} \delta .
$$

Proof. Let $I$ be a maximum independent set in $G$. If every other vertex is adjacent to all of $I$, then $G$ is the join of an independent set and a $K_{r-1}$-free set, so we are done. Thus we may assume that there exists a vertex $s_{1} \in V(G)-I$ such that $I \nsubseteq N\left(s_{1}\right)$ (but by the maximality of $\left.I, N\left(s_{1}\right) \cap I \neq \emptyset\right)$.

Consider some vertex $x \in I$ that is not adjacent to $s_{1}$. As $G$ is a maximal $K_{r}$-free graph, there must be vertices $\left\{s_{2}, \ldots, s_{r-1}\right\}$ in $N\left(s_{1}\right) \cap N(w)$ which induce a clique of order $r-2$. Let $S=\left\{s_{1}, \ldots, s_{r-1}\right\}$, so that $S$ induces a copy of $K_{r-1}$, but $S \cap I=\emptyset$.

At this point, we consider two cases, based on the diversity of neighborhoods of vertices of $I$ in $S$. For a set $S^{\prime} \subset S$, let $N\left(S^{\prime}\right)$ denote the common neighborhood of vertices in $S^{\prime}$.

1. For every subset $S^{\prime} \subseteq S$ of $r-2$ vertices, $N\left(S^{\prime}\right) \cap I \neq \emptyset$.

As each set of $r-2$ vertices in $S$ has a common neighbor in $I$, this forms a sunflower graph $S G_{r}$. To bound the independence number from above, we use double counting on the adjacencies from $S G_{r}$ to the rest of the graph. Each vertex in $G$ can be adjacent to at most $r-2$ of the vertices of $S$ without forming a copy of $K_{r}$. As a result, a vertex in $I$ can be adjacent to at most $r-2$ vertices of $S G_{r}$. Every vertex in $V(G)-I$ can be adjacent to at most $2 r-4$ (either $r-1$ of the vertices in $I$, and $r-3$ vertices in $S$, or at most $r-2$ from both the vertices in $S$ and the vertices of $S G_{r}$ in $\left.I\right)$. As each vertex in $S G_{r}$ has degree $\delta$, we obtain the following:

$$
2(r-1) \delta \leqslant(2 r-4)(n-|I|)+(r-2)|I|=(2 r-4) n-(r-2)|I| .
$$

Solving for $|I|$ yields, $|I| \leqslant 2(n-\delta)-(2 /(r-2)) \delta$.

2. There is a subset $S^{\prime} \subseteq S$ of $k$ vertices $(2 \leqslant k \leqslant r-2), N\left(S^{\prime}\right) \cap I=\emptyset$.

In this case, let $k$ be the smallest such value for which this is true, and let $S^{\prime} \subset S$ be the corresponding set of vertices. Recall that $S$ is disjoint from $I$; by the maximality of $I$, every vertex in $S$ is adjacent to a vertex in $I$, so $k \geqslant 2$. Our goal is to build a set of vertices $S^{\prime \prime \prime}$ which induce a clique of order $k$ and consider the intersection of the neighborhoods of these vertices.

Before determining the set $S^{\prime \prime \prime}$, we introduce the slack of a family of sets, as a convenient tool to clarify the discussion of this case (used for a similar purpose in [GL11]). Consider a family of sets $\mathcal{A}=\left\{A_{1}, A_{2}, \ldots, A_{k}\right\}$ on the set $\{1,2, \ldots, n\}$, and 
let $N_{i}$ denote the pairwise disjoint sets of elements which are contained in exactly $i$ sets. We define the slack of $\mathcal{A}$ is defined to be

$$
\operatorname{Slack}(\mathcal{A})=\sum_{i=0}^{k-2}(k-i-1)\left|N_{i}\right|
$$

We note that the sum of orders of the sets $A_{i}$ can be written as a weighted sum of the orders of the $N_{i}$ sets, that is

$$
\sum_{i=1}^{k}\left|A_{i}\right|=k\left|N_{k}\right|+(k-1)\left|N_{k-1}\right|+(k-2)\left|N_{k-2}\right|+\cdots+\left|N_{1}\right| .
$$

The slack will be used to help determine a bound on $\left|N_{k}\right|$. Isolating one copy of $\left|N_{k}\right|$, we get

$$
\begin{aligned}
\left|N_{k}\right| & =\sum_{i=1}^{k}\left|A_{i}\right|-\left[(k-1)\left|N_{k-1}\right|+(k-2)\left|N_{k-2}\right|+\cdots+\left|N_{1}\right|\right]-(k-1)\left|N_{k}\right|, \\
& =\sum_{i=1}^{k}\left|A_{i}\right|-(k-1)\left(\left|N_{k}\right|+\left|N_{k-1}\right|+\cdots+\left|N_{1}\right|+\left|N_{0}\right|\right)+\operatorname{Slack}(\mathcal{A}) .
\end{aligned}
$$

As the sets $N_{i}$ are pairwise disjoint, $\left|N_{k}\right|+\left|N_{k-1}\right|+\cdots+\left|N_{1}\right|+\left|N_{0}\right|=n$, and we obtain the following equality,

$$
\left|N_{k}\right|=\sum_{i=1}^{k}\left|A_{i}\right|-(k-1) n+\operatorname{Slack}(\mathcal{A})
$$

Now, choose a set $S^{\prime \prime \prime}$ by first choosing a suitable subset $S^{\prime \prime} \subset S^{\prime}$ according to one of the following cases:

(a) Suppose there is a vertex $v \in S^{\prime}$ such that $|N(v) \cap I| \geqslant \frac{k-1}{k}|I|$.

In this case, choose $S^{\prime \prime}=S^{\prime}-\{v\}$. Since $N\left(S^{\prime}\right) \cap I=\emptyset$, each vertex in $N(v) \cap I$ is not adjacent to at least one vertex in $S^{\prime \prime}$. Then, chose a vertex $w \in N\left(S^{\prime \prime}\right) \cap I$ to add to form $S^{\prime \prime \prime}=S^{\prime \prime} \cap\{w\}$. By forming the clique in this way, at least $\frac{k-1}{k}|I|$ vertices are adjacent to at most $k-2$ vertices in $S^{\prime \prime \prime}$. Let $\mathcal{S}$ denote the family of neighborhoods of each of the vertices in $S^{\prime \prime \prime}$. Then,

$$
\operatorname{Slack}(\mathcal{S}) \geqslant \frac{k-1}{k}|I|
$$

(b) Suppose there is no vertex $v \in S^{\prime}$ such that $|N(v) \cap I| \geqslant \frac{k-1}{k}|I|$.

In this case, choose $S^{\prime \prime}$ to be any set of $k-1$ vertices in $S^{\prime}$. Then there are at least $((k-1) / k)|I|$ non-adjacencies between $S^{\prime}$ and $I$. Again, chose a vertex $w \in N\left(S^{\prime \prime}\right) \cap I$ to add to form $S^{\prime \prime \prime}=S^{\prime \prime} \cap\{w\}$, and let $\mathcal{S}$ denote the family of neighborhoods of each of the vertices in $S^{\prime \prime \prime}$. Then,

$$
\operatorname{Slack}(\mathcal{S}) \geqslant \frac{k-1}{k}|I|
$$


Let $U$ denote the common neighborhood of $S^{\prime \prime \prime}$, and note that $U$ must be $K_{s}$-free, where $s=r-k$. Taking into account the slack observed above, we use Equation 1 to obtain

$$
|U| \geqslant k \delta-(k-1) n+\frac{k-1}{k}|I|
$$

To apply the Bootstrapping Lemma, we next consider modest lower bounds on $\alpha(G)$. For $K_{r}$-free graphs which are not the join of an independent set and a $K_{r-1}$-free graph, this serves the dual purposes of

1. demonstrating that the second statement from the Bootstrapping Lemma must be true, and

2. providing a bound on the $K_{r-k}$-free set $\left|U_{s}\right|$ from the second statement.

In particular, in the case $r=4$, modest bounds on $\alpha(G)$ result in improved bounds on $\alpha(G)$ (Hence, this is a "bootstrapping" lemma). In fact, the same holds true for $r>4$ (though not as directly).

\section{4 "Modest" bounds on $\alpha(G)$.}

\section{1 $K_{r}$-free graphs $(r>4)$.}

To provide a "modest" bound on the independence number, we define a function $f=$ $f(r, \delta, n)$ as follows:

$$
f(r, \delta, n)=\delta-\frac{r-3}{r-1} n
$$

This function provides a bound on $\alpha(G)$, and is well-known (for example, see [ES70]). For completeness the following lemma gives a proof of this simple result.

Lemma 7. For any $K_{r}$-free graph $G$

$$
\alpha(G) \geqslant f(r, \delta, n)
$$

Proof. Let $s$ be the minimum value such that $G$ contains a copy of $K_{s-1}$. Choose a copy of $S=K_{s-1}$ in $G$. At least $(s-1) \delta-(s-3) n$ vertices in $G$ are adjacent to $r-2$ vertices in this copy. For any $S^{\prime} \subset S$ of order $s-2, N\left(S^{\prime}\right)$ is independent. Since there are $s-1$ different choices for $S^{\prime}$, then there is some $S^{\prime}$ such that $\left|N\left(S^{\prime}\right)\right| \geqslant \delta-((s-3) /(s-1)) n$, and $N\left(S^{\prime}\right)$ must necessarily be independent. Note that $s \leqslant r$ and $f(s, \delta, n)$ is decreasing in $s$, implying the result.

This bound on $\alpha(G)$ can be used to determine when statement (3) in the Bootstrapping Lemma is not satisfied. Solving, we obtain the following: 
Fact 8 .

$$
f(r, \delta, n) \geqslant 2(n-\delta)-\frac{2}{r-2} \delta \text { for } \delta>\frac{(3 r-5)(r-2)}{(3 r-4)(r-1)}
$$

For $r=5$, the bound on $\delta$ above is $15 / 22=.6 \overline{81}$. In fact, for $r \geqslant 5$, we note that $((5 r-11) /(5 r-5)) n$ is larger than the bound on $\delta$ provided. Therefore, in the results regarding both $r=5$ or $r>5$ described in the introduction, we may assume that statement (3) in the Bootstrapping Lemma is not satisfied.

\section{$4.2 \quad K_{4}$-free graphs.}

For $r=4$, the modest bounds on $\alpha(G)$ are improved in order to apply the bootstrapping lemma effectively for a larger range of values on $\delta$. First, we obtain a simple bound in the case when the neighborhood of a minimum degree vertex is bipartite or has large odd girth.

Lemma 9. Let $G$ be a maximal $K_{4}$-free graph. Suppose $u$ is a vertex of minimum degree. If $C_{5} \nsubseteq \mathbb{N}(u)$, then

$$
\alpha(G) \geqslant \min \left\{\frac{\delta}{2}, \frac{17}{7} \delta-n\right\} .
$$

Proof. Let $G$ be a maximal $K_{4}$-free graph. Consider a minimum degree vertex $u$. If the graph induced by $N(u)$ is bipartite, then one of the bipartitions has order at least $\delta / 2$. Otherwise, the graph induced by $N(u)$ is not bipartite, and does not contain $C_{5}$. In this case, consider the shortest odd cycle $C=C_{2 k+1}$ for some $k>2$.

Each vertex in $N(u)$ can be adjacent to at most 2 vertices on this cycle. As a result, the number of edges from the vertices in $N(u)-C$ to the vertices in $C$ is at most $2(\delta-(2 k+1))$. Adding the degrees of end-vertices over all edges in the cycle $C$ gives twice the number of edges from the vertices in $N(u)-C$ to the vertices in $C$ (minus $2(2 k-1)$, twice the number of edges of the cycle), so there is some edge such that the endvertices are adjacent to at most $(4 /(2 k+1)) \delta$ vertices in $N(u)$. The common intersection of the neighborhoods of the end-vertices of this edge form an independent set. As a result, we obtain

$$
\alpha(G) \geqslant 2 \delta-(n-((2 k-3) /(2 k+1)) \delta) .
$$

This is minimized at $k=3$, and yields $\alpha(G) \geqslant 2 \delta-n+(3 / 7) \delta=(17 / 7) \delta-n$.

Next, we consider more closely the case when $C_{5} \subseteq N(u)$ (where $u$ is a minimum degree vertex), that is, $W_{5}$ (the 5 -wheel) is a subgraph of $G$. We will make use of $W_{5}$ for double counting, a strategy used effectively in a variety of ways by Brandt (e.g. [Bra03]). In order to use double counting, we define the following sets.

$$
X_{i}=\left\{v \in V(G)-N(u):\left|N(v) \cap W_{5}\right|=i\right\} \text { for } i=0,1,2,3,4,5 .
$$


Since there are 6 vertices in $W_{5}$, there are at least $6 \delta$ neighbors of vertices in $W_{5}$. Also, any vertex in $N(u)$ can be adjacent to at most 3 vertices of $W_{5}$. Therefore,

$$
6 \delta \leqslant 3 \delta+\left|X_{1}\right|+2\left|X_{2}\right|+3\left|X_{3}\right|+4\left|X_{4}\right|+5\left|X_{5}\right| .
$$

Since the sets $X_{i}$ partition $V(G)-N(u)$, we can refine this expression in two different ways to obtain the following two bounds.

$$
\begin{aligned}
& 6 \delta \leqslant 3 \delta+2(n-\delta)+\left|X_{3}\right|+2\left|X_{4}\right|+3\left|X_{5}\right|, \\
& 6 \delta \leqslant 3 \delta+3(n-\delta)+\left|X_{4}\right|+2\left|X_{5}\right| .
\end{aligned}
$$

These yield the following two inequalities regarding the cardinalities of $X_{i}$ :

$$
\begin{aligned}
\left|X_{4}\right|+2\left|X_{5}\right| & \geqslant 6 \delta-3 n, \\
\left|X_{3}\right|+2\left|X_{4}\right|+3\left|X_{5}\right| & \geqslant 5 \delta-2 n .
\end{aligned}
$$

Note that the vertices in $X_{5}$ cannot be adjacent to any vertex in $X_{4} \cup X_{3}$, or $K_{4} \subset G$. Since both $X_{4}$ and $X_{5}$ are independent sets,

$$
\left|X_{4}\right|+\left|X_{5}\right| \leqslant \alpha(G)
$$

In the following lemmas, we shall provide bounds on $\alpha(G)$ using the inequalities above, based on the structure of the graph induced by $X_{3} \cup X_{4}$.

Lemma 10. Let $G$ be a maximal $K_{4}$-free graph such that the neighborhood of a vertex of minimum degree contains a copy of $C_{5}$. Then,

$$
\alpha(G) \geqslant g(n, \delta):=\min \left\{\frac{9}{4} \delta-n, \frac{1}{3}(5 \delta-2 n), \frac{5}{2}(2 \delta-n)\right\} .
$$

Proof. To obtain these bounds, we consider different cases based on the graph induced by $X_{3} \cup X_{4}$.

1. The graph induced by $X_{3} \cup X_{4}$ is triangle-free and non-bipartite,

In the set $X_{3} \cup X_{4}$, form a set $S$ such that $X_{4} \subset S$ and $S$ is a maximal independent. Since $X_{3} \cup X_{4}$ is not bipartite, there must be some edge $(u, v)$ such that $u, v \in$ $\left(X_{3} \cup X_{4}\right)-S$ and $|N(u) \cap S|,|N(v) \cap S| \neq \emptyset$. Furthermore, since $X_{3} \cup X_{4}$ is triangle-free, $|N(u) \cap N(v) \cap S|=0$, implying either $|N(u) \cap S|<(1 / 2)|S|$ or $|N(v) \cap S|<(1 / 2)|S|$. Without loss of generality, suppose $|N(u) \cap S|<(1 / 2)|S|$, and consider a vertex $s \in N(u) \cap S$. Neither vertex of the edge $(u, s)$ is adjacent to the vertices in $S-N(u)$ or $X_{5}$, and therefore,

$$
\begin{aligned}
\alpha(G) & \geqslant 2 \delta-n+\left|X_{5}\right|+\frac{1}{2}|S| \\
& \geqslant 2 \delta-n+\frac{1}{2}\left(2\left|X_{5}\right|+\left|X_{4}\right|\right) \\
& \geqslant \frac{5}{2}(2 \delta-n) .
\end{aligned}
$$


2. The graph induced by $X_{3} \cup X_{4}$ contains a triangle.

Each vertex of the triangle in $X_{3} \cup X_{4}$ cannot be adjacent to $X_{5}$. Therefore, we obtain the following bound on $\alpha(G)$ :

$$
\begin{aligned}
\alpha(G) & \geqslant \frac{1}{3}\left(3 \delta-n+\left|X_{5}\right|\right) \\
& \geqslant \frac{1}{3}\left(3 \delta-n+\left|X_{5}\right|\right)+\frac{1}{3}\left(\left|X_{4}\right|+\left|X_{5}\right|\right)-\frac{1}{3} \alpha(G) .
\end{aligned}
$$

Applying Inequality 2 yields the bound $\alpha(G) \geqslant(9 / 4) \delta-n$.

3. The graph induced by $X_{3} \cup X_{4}$ is triangle-free and bipartite.

Since $X_{5}$ is not adjacent to any vertex in $X_{3} \cup X_{4}$,

$$
\begin{aligned}
\alpha(G) & \geqslant \frac{1}{2}\left(\left|X_{3}\right|+\left|X_{4}\right|\right)+\left|X_{5}\right| \\
& =\frac{1}{2}\left(\left|X_{3}\right|+2\left|X_{4}\right|+3\left|X_{5}\right|\right)-\frac{1}{2}\left(\left|X_{4}\right|+\left|X_{5}\right|\right) .
\end{aligned}
$$

Applying Inequalities 2 and 3 yields the bound $\alpha(G) \geqslant(1 / 3)(5 \delta-2 n)$.

The result of this section is the following fact:

Fact 11 .

$$
g(n, \delta) \geqslant 2(n-\delta)-\delta=2 n-3 \delta \text { for } \delta>\frac{4}{7} n .
$$

Now, as in the case with $r \geqslant 5$, we may assume that statement (3) in the Bootstrapping Lemma is not satisfied for our results regarding $r=4$.

\section{Applying the Bootstrapping Lemma}

\section{$5.1 \quad K_{4}$-free graphs}

In the case of $r=4$, applying the Bootstrapping Lemma to prove Theorem 3 is a very simple matter.

Proof of Theorem 3. Suppose that $G$ is a $K_{4}$-free graph on $n$ vertices with $\delta>(4 / 7) n$, such that $G$ cannot be decomposed as the join of an independent set and a triangle-free graph. By the structure of $G$ and Fact 11, both statements (2) and (3) in the Bootstrapping Lemma are not satisfied, implying that there is a set $U_{2}$ which is independent, such that

$$
\alpha(G) \geqslant\left|U_{2}\right| \geqslant 2 \delta-n+\frac{1}{2} \alpha(G) .
$$

Solving for $\alpha(G)$ yields $\alpha(G) \geqslant 2(2 \delta-n)$, and we note that for this bound, $\delta(G) \geqslant(3 / 5) n$ implies $\alpha(G) \geqslant(2 / 5) n>(1 / 3) n$. 
When $G=H \vee I$ is the join of a triangle-free graph $H$ and an independent set $I$ with $\alpha(G)<(1 / 3) n$, it is easy to show that $\delta<(3 / 5) n$, completing an alternative proof of Theorem 2 (for the case $r=4$ ). It must be the case that $|H|>(2 / 3) n$, where $n$ is the number of vertices in $G$, or $\alpha(G) \geqslant|I| \geqslant(1 / 3) n$. As a consequence, $H$ must also not be bipartite, implying that the minimum degree of $H$ is at most $(2 / 5)|V(H)|$. Considering the degree in such a graph, we get

$$
\delta \leqslant(n-|H|)+\frac{2}{5}|H|=n-\frac{3}{5}|H| .
$$

This is maximized at the minimum value of $|H|$ (and $|H|>(2 / 3) n$ ) implying that the graph $G$ must have minimum degree less than $(3 / 5) n$.

\section{$5.2 \quad K_{5}$-free graphs}

The approach for $K_{5}$-free graphs is dependent on the structure. Graphs which can be written as the join of an independent set and a $K_{4}$-free set, or as the join of two trianglefree graphs are treated separately. However, graphs which cannot be written as the join of an independent set and a $K_{4}$-free set, or as the join of two triangle-free graphs have large independent sets, and this is demonstrated by the proof of Theorem 4, given below.

Proof of Theorem 4. For $\delta>(15 / 22) n$, the bootstrapping lemma, combined with the structure of $G$ and the modest bounds on $\alpha(G)$ for $K_{5}$-free graphs, yields a set $U_{k}$ for either $k=2$ or $k=3$, such that the set is $K_{5-k}$-free and

$$
\left|U_{k}\right| \geqslant k \delta-(k-1) n+\frac{k-1}{k} \alpha(G) .
$$

For $k=3$, the set $U_{3}$ is an independent set. Using $\alpha(G) \geqslant\left|U_{3}\right|$ and substituting $k=3$ into Equation 5, the bound $\alpha(G) \geqslant 3(3 \delta-2 n)$ is obtained.

For $k=2$, the set $U_{2}$ induces a triangle-free graph, and certainly $\left|U_{2}\right|$ is at most as large as the order of the largest induced triangle-free subgraph (say $S$ ). If the largest induced triangle-free subgraph is bipartite, then applying $2 \alpha(G) \geqslant|S| \geqslant\left|U_{3}\right|$ and substituting $k=3$ into Equation 5, yields the bound $\alpha(G) \geqslant(2 / 3)(2 \delta-n)$.

In the event that $S$ is not bipartite, there is some edge $(s, t) \in S$, such that both end-vertices of the edge are not adjacent to a common set of at least $(1 / 5)|S|$ vertices in $S$ (by the same reasoning as in Lemma 9). The intersection of the neighborhoods of $s$ and $t$ is again a triangle-free graph, so we obtain

$$
|S| \geqslant 2 \delta-n+\frac{1}{5}|S|
$$

Solving, we obtain $|S| \geqslant(5 / 4)(2 \delta-n)$. In fact, this yields two disjoint triangle-free sets $(S$ and the common neighborhood of $s$ and $t$ ), both of order at least $(5 / 4)(2 \delta-n)$. Therefore, we may choose $S_{1}$ and $S_{2}$ to be disjoint sets such that $S_{1}$ and $S_{2}$ both induce triangle-free graphs, and both have order at least $(5 / 4)(2 \delta-n)$, such that the union of $S_{1}$ 
and $S_{2}$ is maximal. That is, any vertex $v \in V(G)-\left(S_{1} \cup S_{2}\right)$ cannot be added to either $S_{1}$ or $S_{2}$ without creating a triangle. Equivalently, each vertex $v \in V(G)-\left(S_{1} \cup S_{2}\right)$ is adjacent to both vertices of an edge $\left(u_{1}, u_{2}\right) \in S_{1}$ and an edge $\left(w_{1}, w_{2}\right) \in S_{2}$. For these vertices, we bound the degrees in terms of $\alpha(G),\left|S_{1}\right|$, and $\left|S_{2}\right|$.

1. $S_{1}$ and $S_{2}$ both induce triangle-free graphs, implying

$$
\begin{aligned}
& \left|N\left(u_{1}\right) \cap S_{1}\right|+\left|N\left(u_{2}\right) \cap S_{1}\right| \leqslant 2 \alpha(G), \text { and } \\
& \left|N\left(w_{1}\right) \cap S_{2}\right|+\left|N\left(w_{2}\right) \cap S_{2}\right| \leqslant 2 \alpha(G) .
\end{aligned}
$$

2. The common intersection of neighborhoods of the vertices of a triangle must be independent, implying

$$
\begin{aligned}
& \left|N\left(u_{1}\right) \cap S_{2}\right|+\left|N\left(u_{2}\right) \cap S_{2}\right|+\left|N(v) \cap S_{2}\right| \leqslant 2\left|S_{2}\right|+\alpha(G), \text { and } \\
& \left|N\left(w_{1}\right) \cap S_{1}\right|+\left|N\left(w_{2}\right) \cap S_{1}\right|+\left|N(v) \cap S_{1}\right| \leqslant 2\left|S_{1}\right|+\alpha(G) .
\end{aligned}
$$

3. Trivially, for any vertex $x, \mid N(x) \cap\left(V(G)-\left(S_{1} \cup S_{2}\right) \mid \leqslant n-\left(\left|S_{1}\right|+\left|S_{2}\right|\right)\right.$.

Therefore, summing the degrees of the vertices $v, u_{1}, u_{2}, w_{1}$, and $w_{2}$, we obtain

$$
\begin{aligned}
5 \delta & \leqslant 4 \alpha(G)+2\left|S_{1}\right|+2\left|S_{2}\right|+2 \alpha(G)+5\left(n-\left|S_{1}\right|-\left|S_{2}\right|\right) \\
& \leqslant 5 n-3\left|S_{1}\right|-3\left|S_{2}\right|+6 \alpha(G) .
\end{aligned}
$$

Applying the lower bound on $\left|S_{1}\right|$ and $\left|S_{2}\right|$ and solving for $\alpha(G)$, we obtain

$$
\alpha(G) \geqslant \frac{1}{6}\left(20 \delta-\frac{25}{2} n\right) .
$$

At this point, we proceed to consider graphs that can be written as the join of a $K_{4}$-free graph and an independent set.

Lemma 12. Let $G$ be a maximal $K_{5}$-free graph, such that $G$ is the join of a $K_{4}$-free graph and an independent set, and $G$ is not the join of two triangle-free graphs. Then,

$$
\alpha(G) \geqslant \frac{2}{3}(2 \delta-n)
$$

Proof. Let $G$ be the join of a $K_{4}$-free graph $H$ and an independent set. Note that since $G$ is not the join of a two triangle-free graphs, then the $H$ cannot be the join of a triangle-free graph and an independent set. Therefore, there is an independent set in $H$ of order

$$
\alpha(G) \geqslant 2(2(\delta-\alpha(G))-(n-\alpha(G))) .
$$

This yields $\alpha(G) \geqslant(2 / 3)(2 \delta-n)$.

For a graph that is the join of two triangle-free graphs, results regarding the structure of triangle-free graphs with large minimum degree can be used. 
Lemma 13. Let $G$ be a maximal $K_{5}$-free graph, such that $G$ is the join of two triangle-free graphs. Then,

$$
\alpha(G) \geqslant \begin{cases}\frac{1}{4} n & \text { for } \delta>\frac{7}{10} n \\ \frac{1}{5} n & \text { for } \delta>\frac{11}{16} n\end{cases}
$$

Proof. Suppose that $G$ is the join of triangle-free graphs $H_{1}$ and $H_{2}$. We may choose $H_{1}$ to be the larger. The relative degree of a vertex in $H_{1}$ is at least $\left(\delta-\left(n-\left|H_{1}\right|\right)\right) /\left|H_{1}\right|$. This can be seen to be increasing in $H_{1}$, so we choose $H_{1}=(1 / 2) n$ to minimize the relative degree, yielding a relative degree of $2(\delta / n)-1$.

When the relative degree is larger than $(2 / 5)$, the graph $H_{1}$ must be bipartite. This occurs at $\delta=(7 / 10) n$. When the relative degree is larger than $(3 / 8)$, the graph $H_{1}$ is either bipartite or homomorphic to a 5 -cycle. This occurs at $\delta=(11 / 16) n$.

Both of the thresholds in Lemma 13 are sharp, as seen by the join of two 5-cycles, and the join of two copies of the Wagner graph, the Möbius ladder on 8 vertices.

Define the function $g(n, \delta)$, by the following:

$$
h(n, \delta):=\min \left\{3(3 \delta-2 n), \frac{1}{6}\left(20 \delta-\frac{25}{2} n\right), \frac{2}{3}(2 \delta-n)\right\} .
$$

To achieve the proof of Corollary 5 , it is enough to note that $31 / 45>11 / 16$, and for $\delta \geqslant(31 / 45) n, h(n, \delta) \geqslant(1 / 5) n$.

\section{$5.3 \quad K_{r}$-free $(r>5)$}

In this section, we provide the proof of Theorem 6. In order to present the proof of the following lemma more smoothly, let

$$
\delta_{r}=\frac{r-3}{r-2}+\frac{1}{(r-2)^{2}(r-1)} .
$$

To begin with, we show that there must be a large $K_{s}$-free set in a $K_{r}$-free graph with $\delta>\delta_{r} n$.

Lemma 14. Let $G$ be a $K_{r}$-free graph with $\delta(G) \geqslant \delta_{r} n$ that cannot be written as the join of a $K_{r-1}$-free graph and an independent set. Then there is some set $S$ which is $K_{s}$-free $(s<r)$ such that

$$
|S| \geqslant \frac{s-1}{r-1} n
$$

Proof. The bootstrapping lemma guarantees a set $U_{k}$ which is $K_{r-k}$-free, such that

$$
\left|U_{k}\right| \geqslant k \delta-(k-1) n+\frac{k-1}{k} \alpha(G) .
$$

To show this result, we first show it for the particular cases $k=r-2, k=r-3$, and $k=r-4$, and then for $k>r-4$. 


\section{Suppose $k=r-2$.}

If this is the case, $U_{r-2}$ is independent, and in particular $\alpha(G) \geqslant\left|U_{r-2}\right|$. Applying this to Equation 6 allows one to solve for $\alpha(G)$ and obtain $\alpha(G) \geqslant(r-$ 2) $((r-2) \delta-(r-3) n)$. This bound gives $\alpha(G) \geqslant(1 /(r-1)) n$ for $\delta \geqslant \delta_{r} n$.

At this point, it is worth noting that the case $k=r-2$ determined the choice of $\delta_{r}$. For the remaining cases, it is possible to use a slightly smaller (and cleaner) value on $\delta$ than $\delta_{r} n$. In particular, we use

$$
\delta \geqslant \delta_{r} n>\frac{r-3}{r-2} n
$$

The following two bounds for quantities in Equation 6 (using $\delta_{r}>(r-3) /(r-2)$ ) are also used:

$$
\begin{aligned}
k \delta-(k-1) n & >\frac{r-2-k}{r-2} n, \\
\alpha(G) & >f(r, \delta, n)>\frac{r-3}{(r-2)(r-1)}, \\
& >\frac{k}{k-1}\left(\frac{k}{(r-2)(r-1)}\right) \text { for } k<r-4 .
\end{aligned}
$$

2. Suppose $k=r-3$.

First, suppose that $\alpha(G) \geqslant((r-3) /(r-4))\left(\left|U_{r-3}\right|-(n-\delta)\right)$. Then, when substituted into Equation 6, this yields

$$
\left|U_{r-3}\right| \geqslant(r-2) \delta-(r-3) n+\left|U_{r-3}\right| .
$$

However, this is a contradiction, as $\delta_{r} n>(r-3) /(r-2) n$. Therefore, we may assume that $\alpha(G)<((r-3) /(r-4))\left(\left|U_{r-3}\right|-(n-\delta)\right)$. Additionally, since $U_{r-3}$ induces a triangle-free graph, then $\left|N(v) \cap U_{r-3}\right| \leqslant \alpha(G)$. Since this implies that vertices in $U_{r-3}$ may not have many neighbors in $U_{r-3}$, we look at the intersection of the neighborhoods of two adjacent vertices $u, v \in U_{r-3}$ that occurs outside of $U_{r-3}$.

$$
\begin{aligned}
|N(u) \cap N(v)| & \geqslant 2\left(\delta-\frac{r-3}{r-4}\left(\left|U_{r-3}\right|-(n-\delta)\right)\right)-\left(n-\left|U_{r-3}\right|\right), \\
& \left.>2 \delta-2\left(\frac{r-3}{r-4}\right)\left(\left|U_{r-3}\right|+(n-\delta)\right)-n+\left|U_{r-3}\right|\right) .
\end{aligned}
$$

Since $(r-3) /(r-4)$ attains its maximum at $r=6$ (when restricted to $r \geqslant 6$ ), we can simplify this to the following:

$$
\begin{aligned}
|N(u) \cap N(v)| & \left.>2 \delta-3\left(\left|U_{r-3}\right|+(n-\delta)\right)-n+\left|U_{r-3}\right|\right) \\
& =2 n-\delta-2\left|U_{r-3}\right| .
\end{aligned}
$$


We note that $\delta \leqslant(r-2) /(r-1) n$ for a $K_{r}$-free graph. Thus, either $\left|U_{r-3}\right| \geqslant$ $(2 /(r-1)) n$, or

$$
|N(u) \cap N(v)| \geqslant \frac{r-3}{r-1} n .
$$

As $N(u) \cap N(v)$ does not contain a copy of $K_{r-2}$, either of these outcomes implies the result.

3. Suppose $k=r-4$.

In this case, $U_{r-4}$ is $K_{4}$-free. We will let $S$ be a set of vertices of largest order which induce a $K_{4}$-free graph (so $\left.|S| \geqslant\left|U_{r-4}\right|\right)$. First, we will show that it must be possible to partition $S$ into an independent set and a triangle-free graph. If not, and $S$ induces a $K_{4}$-free graph that cannot be partitioned into a triangle-free graph and an independent set, then Theorem 3 implies a large independent set. In particular, each vertex in $S$ must have at least $\delta-(n-|S|)$ neighbors in $S$, so

$$
\alpha(G) \geqslant 2(2(\delta-(n-|S|))-|S|)=2|S|-4(n-\delta) .
$$

Since $|S| \geqslant\left|U_{r-4}\right| \geqslant(r-4) \delta-(r-5) n$, and $\delta \geqslant \delta_{r} n \geqslant(r-3) /(r-2) n$, then the quantity $2|S|-4(n-\delta)>0$. Using the bound on $\left|U_{r-4}\right|$ and $|S| \geqslant\left|U_{r-4}\right|$, we get

$$
|S| \geqslant(r-4) \delta-(r-5) n+\frac{r-5}{r-4}(2|S|-4(n-\delta)) .
$$

Using $r \geqslant 6$ in the fraction $(r-5) /(r-4)$, we can simplify this to the following:

$$
|S| \geqslant(r-2) \delta-(r-3) n+|S| .
$$

However, this would imply that $\delta<(r-2) /(r-3) n$, a contradiction. Therefore, we may assume $S$ can be partitioned into an independent set and a triangle-free graph.

In this case, we may suppose the order of the triangle-free graph is less than $(2 /(r-$ 1)) $n$ (or we would be done). Therefore,

$$
\alpha(G) \geqslant|S|-\frac{2}{r-1} n \geqslant(r-4) \delta-(r-5) n+\frac{r-5}{r-4} \alpha(G)-\frac{2}{r-1} n .
$$

Solving for $\alpha(G)$, this yields

$$
\alpha(G) \geqslant(r-4)\left((r-4) \delta-(r-5) n-\frac{2}{r-1} n\right) .
$$

Finally, applying Equation 9 yields the following inequality (for $r \geqslant 6$ ),

$$
\alpha(G) \geqslant(r-4)\left(\frac{2}{r-2}-\frac{2}{r-1}\right)=\frac{2 r-8}{r-2}\left(\frac{1}{r-1}\right) \geqslant \frac{1}{r-1} .
$$


4. Suppose $k<r-4$.

In this case, simply apply Equations 9 and 7 to the bound on $\left|U_{k}\right|$ given by the bootstrapping lemma.

$$
\begin{aligned}
\left|U_{k}\right| & \geqslant k \delta-(k-1) n+\frac{k-1}{k} \alpha(G) \\
& \geqslant\left(\frac{r-k-2}{r-2}+\frac{k}{(r-2)(r-1)}\right) n \\
& =\left(\frac{r-k-1}{r-1}\right) n
\end{aligned}
$$

Now we are ready to prove the main result by adding in the induction step.

Proof of Theorem 6. In the event that $G$ can be written as the join of a $K_{r-1}$-free graph $S$ and an independent set $I$, than $|I|>(1 /(r-1)) n$, and we are done, or $|S|>((r-2) /(r-$ 1))n. If $G$ cannot be written as the join of a $K_{r-1}$-free graph and an independent set, then Lemma 14 guarantees that there is a $K_{s}$-free set $S$ such that $|S| \geqslant \frac{s-1}{r-1} n$. Therefore, in either case, we want to use induction applied to the set $S$. If there is an independent set of order $(1 /(s-1))|S|$ contained in $S$, then this implies an independent set of order $(1 /(r-1)) n$ in $G$. Therefore, since the cases $r=4$ and $r=5$ provide base cases, it only remains to be shown that a minimum degree greater than $\delta_{r} n$ implies that the degree of a vertex in $S$ is at least $\delta_{s}|S|$.

For a vertex $u \in S$, we can write

$$
\frac{|N(u) \cap S|}{|S|} \geqslant \frac{\delta-(n-|S|)}{|S|}=1-\frac{n-\delta}{|S|} .
$$

As $n-\delta>0$, we can use the lower bound from Lemma 14, as well as $\delta>\delta_{r} n$, to get

$$
\begin{aligned}
1-\frac{n-\delta}{|S|} & \geqslant 1-\frac{r-1}{s-1}\left(\frac{1}{r-2}-\frac{1}{(r-2)^{2}(r-1)}\right) \\
& =1-\frac{r-1}{(r-2)(s-1)}+\frac{1}{(r-2)^{2}(s-1)} .
\end{aligned}
$$

At this point, we note that the expression above decreases in $r$, and certainly $r>s$. Therefore,

$$
1-\frac{n-\delta}{|S|} \geqslant 1-\frac{s-1}{(s-2)(s-1)}+\frac{1}{(s-2)^{2}(s-1)}=\delta_{s}
$$

\section{References}

$\left[\mathrm{ABG}^{+} 13\right]$ P. Allen, J. Böttcher, S. Griffiths, Y. Kohayakawa, and R. Morris. The chromatic thresholds of graphs. Adv. Math., 235:261-295, 2013. 
[AES74] B. Andrásfai, P. Erdős, and V. T. Sós. On the connection between chromatic number, maximal clique and minimal degree of a graph. Discrete Math., 8:205218, 1974.

[BE76] B. Bollobás and P. Erdős. On a Ramsey-Turán type problem. J. Combinatorial Theory Ser. B, 21(2):166-168, 1976.

[Bra01] S. Brandt. Dense graphs with bounded clique number. German habilitation thesis, Freie Universität Berlin, 2001.

[Bra03] S. Brandt. On the structure of graphs with bounded clique number. Combinatorica, 23(4):693-696, 2003.

[BTar] S. Brandt and S. Thomassé. Dense triangle-free graphs are four colorable: A solution to the Erdös-Simonovits problem. J. Combin. Theory: Ser. B., to appear.

[ES70] P. Erdős and V. T. Sós. Some remarks on Ramsey's and Turán's theorem. In Combinatorial theory and its applications, II (Proc. Colloq., Balatonfüred, 1969), pages 395-404. North-Holland, Amsterdam, 1970.

[GL11] W. Goddard and J. Lyle. Dense graphs with small clique number. J. Graph Theory, 66(4):319-331, 2011.

[Euc06] T. Łuczak. On the structure of triangle-free graphs of large minimum degree. Combinatorica, 26(4):489-493, 2006.

[Sze72] E. Szemerédi. On graphs containing no complete subgraph with 4 vertices. Mat. Lapok, 23:113-116 (1973), 1972.

[Tho02] C. Thomassen. On the chromatic number of triangle-free graphs of large minimum degree. Combinatorica, 22(4):591-596, 2002.

[Tur41] P. Turán. Eine Extremalaufgabe aus der Graphentheorie. Mat. Fiz. Lapok, 48:436-452, 1941. 